\title{
TASA DE REINGRESO NO PROGRAMADO EN CIRUGÍA COLORRECTAL ELECTIVA*
}

\author{
Drs. Guillermo Bannura C. ${ }^{1}$, Cristián Gallardo V. ${ }^{1}$, Claudio Vargas R. ${ }^{1}$, \\ Alejandro Barrera E. ${ }^{1}$, Carlos Melo L. ${ }^{1}$, Felipe Illanes F. ${ }^{1}$ \\ 1 Servicio y Departamento de Cirugía Hospital Clínico San Borja Arriarán Campus Centro, \\ Facultad de Medicina Universidad de Chile. \\ Santiago, Chile.
}

\section{Abstract \\ Hospital readmission rates in complex colorrectal surgery}

Background: Non programmed hospital readmission rates are a quality indicator of colorectal surgery. Aim: To analyze the causes of readmission of patients subjected to surgical procedures including intestinal anastomoses. Material and Methods: Analysis of a database of patients subjected to elective intestinal anastomoses in a period of 10 years. All non-programmed readmissions that occurred within 30 days after patient discharge were analyzed. Results: Overall non-programmed readmission rate was $7 \%$ and it was due to medical causes in $55 \%$ of patients. Nine percent of readmitted patients required a new surgical intervention. The figure among patients readmitted due to surgical causes, was $20 \%$. Sixty one percent of patients were admitted at less than six days after discharge and $84 \%$ at less than 10 days. A non-programmed readmission duplicated the total hospitalization lapse and triplicated the rates of new surgical procedures. Conclusions: In this series of patients, the only predictor of a non-programmed readmission was the need for reoperation during the first admission.

Key words: Readmission, colorrectal surgery, intestinal anastomoses.

\section{Resumen}

Antecedentes: La readmisión no programada de un paciente operado es un evento frecuente en la práctica quirúrgica y se considera un indicador de calidad de la atención. El objetivo de este estudio es revisar las causas relevantes de reingreso en nuestro medio, establecer una tasa (TR) que permita una comparación prospectiva de los resultados y, eventualmente, identificar factores de riesgo modificables. Pacientes y Método: Se incluyen todos los pacientes sometidos a cirugía mayor electiva con una anastomosis intestinal en un período de 10 años. Se define como readmisión la re-hospitalización no planificada en el período de 30 días a contar del alta del paciente categorizada como causa médica o quirúrgica. Para el análisis estadístico se empleó el test de regresión logística. Resultados: La TR en la serie fue 7\% (56/791), el 55\% son por causa médica. La

*Recibido el 30 de noviembre de 2014 y aceptado para publicación el 23 de diciembre de 2014.

Los autores no refieren conflictos de interés.

Correspondencia: Dr. Guillermo Bannura C.

gbannura@gtdmail.com 
tasa de re-operación global durante el reingreso fue $9 \%(5 / 56)$, cifra que se eleva al $20 \%(5 / 25)$ en el grupo con alguna causa quirúrgica de re-admisión. El 61\% de los pacientes reingresan antes de los 6 días del egreso y el $84 \%$ antes de los diez días. Un reingreso no planificado duplica el tiempo total de hospitalización ( 9 vs 19 días; $\mathrm{p}=0,001)$ y casi triplica la tasa de reoperación $(\mathrm{p}=0,001)$. Conclusión: En nuestra serie el único factor de riesgo de un reingreso fue el antecedente de una reoperación durante la cirugía índice. La TR es un indicador complejo y los factores predictivos de una re-hospitalización son motivo de controversia.

Palabras clave: Tasa de readmisión, cirugía colorrectal electiva.

\section{Introducción}

En la actualidad la tasa de readmisión (TR) no programada al hospital de un paciente que cursa el postoperatorio inmediato se considera un buen indicador de la calidad y seguridad de la cirugía. Aunque la información de este aspecto crucial en la evolución clínica es escasa en la literatura médica, se estima que una readmisión representa un indicador negativo del cuidado que se asocia a complicaciones de diverso grado, costos no despreciables y un resultado final inadecuado para el estándar ${ }^{1}$. La cirugía mínimamente invasiva (cirugía laparoscópica) y los protocolos de rehabilitación multimodal perioperatoria (fast-track) en cirugía colorrectal electiva han potenciado la estadía acortada y el alta precoz como una forma efectiva de reducir los costos de la cirugía y mejorar la calidad de vida de los pacientes, lo que teóricamente podría afectar la TR no programados ${ }^{2}$. Datos provenientes de series de una institución específica sitúan la TR entre $9 \%$ y el $15 \%$ luego de cirugía colorrectal electiva ${ }^{3,4}$. Un estudio poblacional basado en los datos del SEER en USA sobre 149.622 pacientes en un período de 20 años muestra una TR promedio de $11 \%$ (extremos 10,2-13,7), con un incremento sostenido en el tiempo, mientras que se aprecia una reducción de la estadía hospitalaria en la cirugía primaria en el mismo período (promedio 11,7 días; extremos 14-10,6) . Otro estudio poblacional proveniente del Johns Hopkins School of Public Health estima esta cifra en $11,4 \%$, es decir, 1.239 pacientes de un universo de 10.882 pacientes sometidos a cirugía colorrectal ${ }^{6}$.

El objetivo de este estudio es explorar la incidencia, las causas y el intervalo de reingreso, así como, el impacto clínico en una población de pacientes sometidos en forma consecutiva a cirugía colorrectal mayor electiva en la cual se haya efectuado al menos una anastomosis intestinal. Secundariamente, se intenta identificar factores pronósticos de una readmisión no programada en dichos pacientes.

\section{Pacientes y Método}

Los datos fueron obtenidos de una base de datos prospectiva de cirugía colorrectal electiva de los pacientes sometidos a una resección mayor y/o a una anastomosis intestinal en un período de 10 años en forma consecutiva. Se define como readmisión la rehospitalización no planificada en el período de 30 días a contar del alta del paciente. El grupo de estudio incluye a los pacientes que fueron rehospitalizados por cualquier causa en el lapso de 1 mes luego del egreso. Se revisan los antecedentes clínicos y demográficos de los pacientes reingresados (grupo B), los que se comparan con un grupo control constituido por aquellos pacientes intervenidos en el mismo período que no requirieron de una readmisión en el plazo señalado (grupo A). Se incluye para el análisis los pacientes operados por vía laparoscópica que representan el $10 \%$ de la serie y que fueron intervenidos en los últimos 3 años el período.

Se categoriza el motivo de reingreso como médica o quirúrgica, se mide el intervalo entre el alta y la readmisión y el manejo clínico de los pacientes afectados. Se define como causa quirúrgica de readmisión cualquier evento relacionado directamente con la cirugía realizada y como causa médica el resto. El impacto clínico de esta readmisión se midió por el tiempo de hospitalización, la tasa de infección del sitio operatorio, la tasa de reoperación, la tasa de dehiscencia de la anastomosis y la mortalidad ocurrida hasta 2 meses desde el reingreso, datos que se comparan con la hospitalización índice.

Para comparar ambos grupos se utilizó el test de Chi cuadrado para variables categóricas y el test Mann-Withney y Wilcoxon para variables continuas, considerando significativo un $p<0,05$. Para el análisis de los posibles factores de riesgo de una readmisión no programada se utilizó el test de regresión logística. Los análisis fueron efectuados mediante el sistema computacional STATA 10.

\section{Resultados}

En el período de 10 años señalado se intervinieron en forma electiva 791 pacientes en forma consecutiva en los cuales se practicó al menos una anastomosis, de los cuales $56(7,08 \%)$ requieren una readmisión en los 30 días siguientes luego del egreso médico. Las características demográficas, la patología de base y la intervención realizada se detallan en las Tablas 1 a 3, comparando el grupo A sin reingreso con el grupo B en estudio. Algunos datos 
Tabla 1. Características clínicas y demográficas comparativas

\begin{tabular}{|c|c|c|c|c|c|c|}
\hline \multicolumn{2}{|l|}{ Variable } & \multirow{2}{*}{\multicolumn{2}{|c|}{$\begin{array}{c}\text { No-reingreso } \\
\quad \mathbf{n}=\mathbf{7 3 5} \\
60,5(15-88)\end{array}$}} & \multicolumn{2}{|c|}{$\begin{array}{c}\text { Reingreso } \\
n=56\end{array}$} & \multirow{2}{*}{$\begin{array}{c}\mathbf{p} \\
0,676\end{array}$} \\
\hline Edad promedio & (extremos) años & & & 57,3 & $(19-86)$ & \\
\hline Género femenino & n $(\%)$ & 412 & $(56)$ & 33 & (44) & 0,662 \\
\hline Comorbilidad & $\mathrm{n}(\%)$ & 499 & (67) & 34 & $(60,7)$ & 0,865 \\
\hline Albúmina promedio & $\mathrm{g} / \mathrm{dl}$ (extremos) & & $-5,8)$ & 4,1 & $(2,8-5,8)$ & 0,893 \\
\hline Hematocrito promedio & $\%($ extremos $)$ & 36 , & $4-45)$ & 37 & $(13-49)$ & 0,596 \\
\hline Patología maligna & $\mathrm{n}(\%)$ & 521 & (70) & 37 & $(66)$ & 0,578 \\
\hline
\end{tabular}

Tabla 2. Patología de base en ambos grupos

\begin{tabular}{|lcccc|}
\hline Patología & & $\begin{array}{c}\text { No reingreso } \\
\mathbf{n}=\mathbf{7 3 5}\end{array}$ & $\begin{array}{c}\text { Reingreso } \\
\mathbf{n}=\mathbf{5 6}\end{array}$ & p \\
\hline Cáncer de colon & $\mathrm{n}(\%)$ & $349(47,4)$ & $21(38)$ & 0,351 \\
Cáncer de recto & $\mathrm{n}(\%)$ & $160(21,7)$ & $16(29)$ & 0,226 \\
Enfermedad diverticular & $\mathrm{n}(\%)$ & $81(11)$ & $6(11)$ & 0,377 \\
Megacolon & $\mathrm{n} \mathrm{( \% )}$ & $33(4,5)$ & $3(5)$ & 0,975 \\
EEII* & & $42(5,7)$ & $6(11)$ & 0,737 \\
Misceláneas & $\mathrm{n} \mathrm{( \% )}$ & $70(9,5)$ & $4(7)$ & 0,045 \\
\hline
\end{tabular}

EEII: enfermedad inflamatoria intestinal.

Tabla 3. Operaciones realizadas

\begin{tabular}{|lccc|}
\hline Intervención & $\begin{array}{c}\text { No reingreso } \\
\mathbf{n}=\mathbf{7 3 5}\end{array}$ & $\begin{array}{c}\text { Reingreso } \\
\mathbf{n}=\mathbf{5 6}\end{array}$ & Valor p \\
\hline Resección anterior n (\%) & $323(43,9)$ & $23(41)$ & 0,081 \\
Reconstitución Hartmann n (\%) & $71(9,7)$ & $6(11)$ & 0,358 \\
Colectomía derecha n (\%) & $191(25,9)$ & $14(25)$ & 0,278 \\
Colectomía total + IRA* n (\%) & $25(3,4)$ & $1(2)$ & 0,857 \\
EEA* n (\%) & $101(13,7)$ & $7(13)$ & 0,507 \\
Pouch ileal n (\%) & $24(3,3)$ & $5(9)$ & 0,541 \\
\hline
\end{tabular}

IRA: ileorectoanastomosis. EEA: entero-enteroanastomosis (cierre ileostomía).

comparativos de la operación índice y el impacto clínico del reingreso se aprecia en la Tabla 4, apreciándose que en el grupo que requiere una readmisión el tiempo global de estadía se duplica (9 vs 19 días; $\mathrm{p}=$ $0,001)$, es mayor la tasa de infección del sitio operatorio (ISO) superficial y la tasa de dehiscencia anastomótica (tendencia que no alcanza significación estadística), mientras que la tasa de reoperación casi se triplica en el grupo B $(p=0,001)$. Al analizar las causas de re-hospitalización se aprecia que el 55\% son por causa médica y que la tasa de reoperación global es $9 \%(5 / 56)$, cifra que se eleva al 20\% (5/25) en el grupo con alguna complicación quirúrgica (Tabla 5). El promedio de días egresado antes de la re-hospitalización fue de 5,9 días (extremos 1-15). El intervalo entre el egreso y la readmisión fue de 3 días en el 37,5\% de los pacientes, 6 días en el $61 \%$ y 10 días en el $84 \%$ de los casos. 
Tabla 4. Resultados comparativos de la operación índice

\begin{tabular}{|llccc|}
\hline Variable & & $\begin{array}{c}\text { No-reingreso } \\
\mathbf{n}=\mathbf{7 3 5}\end{array}$ & $\begin{array}{c}\text { Reingreso } \\
\mathbf{n}=\mathbf{5 6}\end{array}$ & Valor p \\
\hline Tiempo operatorio promedio (extremos) min & $121,7(30-400)$ & $129(45-300)$ & 0,332 \\
\hline Hospitalización promedio & $($ extremos) días & $9,42(2-148)$ & $9,39(2-45)$ & 0,218 \\
ISO & $\mathrm{n}(\%)$ & $66(9)$ & $9(16)$ & 0,078 \\
Tasa reoperación & $(\%)$ & 6,1 & 16 & 0,001 \\
Fístula & $\mathrm{n}(\%)$ & $44(5,9)$ & $9(16)$ & 0,059 \\
Mortalidad operatoria & $\mathrm{n}(\%)$ & $7(0,9)$ & 0 & $\mathrm{~ns}$ \\
\hline
\end{tabular}

ISO: infección del sitio operatorio (superficial).

Tabla 5. Causas de readmisión $n=56$

\begin{tabular}{|lrr|}
\hline Médicas & $\mathrm{n}=31$ \\
Síndrome febril & 9 & \\
Deshidratación & 17 & \\
Neumonía intrahospitalaria & 2 & \\
Trombosis venosa profunda & 2 & \\
Lipotimia & 1 & \\
Quirúrgicas & 25 (Reop) \\
ISO superficial & 8 & \\
Fístula enterocutánea & 6 & \\
Obstrucción intestinal & 5 & $(2)$ \\
Colección subfrénica & 3 & $(1)$ \\
Peritonitis (dehiscencia anastomosis) & 1 & $(1)$ \\
Trombosis mesentérica segmentaria & 1 & $(1)$ \\
Manejo ileostomía & 1 & \\
\hline
\end{tabular}

Tabla 6. Factores de riesgo de readmisión (30 días): Regresión logística

\begin{tabular}{|lccc|}
\hline Variables & RD & IC 95\% & p \\
Edad $>$ 70 años & 3,59 & $0,34-4,27$ & 0,216 \\
Género femenino & 1,27 & $0,70-2,30$ & 0,418 \\
\hline Patología benigna & 0,27 & $0,04-1,77$ & 1,175 \\
Comorbilidades & 1,25 & $0,66-2,37$ & 0,487 \\
Albúmina $>3,5 \mathrm{~g} / \mathrm{dl}$ & 0,91 & $0,37-2,20$ & 0,831 \\
Hematocrito $>30 \%$ & 0,96 & $0,43-2,18$ & 0,929 \\
ISO* superficial & 0,66 & $0,25-1,69$ & 0,383 \\
Fístula/dehiscencia* & 1,69 & $0,23-2,11$ & 0,523 \\
Tiempo operatorio $>$ & 0,96 & $0,39-2,37$ & 0,925 \\
180 min & & & \\
Reoperación & 2,91 & $0,10-3,23$ & 0,021 \\
\hline
\end{tabular}

ISO: infección de sitio operatorio. *Fístula y/o dehiscencia de la anastomosis. RD: razón de disparidad. IC: intervalo de confianza.
La re-hospitalización por causa médica fue de 7,3 días como promedio (extremos 1-35), mientras que por causa quirúrgica el promedio fue 12,1 días (extremos 2-43) $(\mathrm{p}=0,05)$. Entre las causas médicas de reingreso destaca el síndrome febril, que en 7 casos se debió a una infección urinaria tratada con antibióticos y en 2 pacientes no se pudo encontrar la causa, cediendo espontáneamente. La deshidratación se debió a una diarrea en 3 pacientes, vómitos en 1 caso, disfunción ileostómica en 2 casos y aporte oral insuficiente en 11. En 2 pacientes este cuadro se complicó con una insuficiencia renal aguda. La infección del sitio operatorio superficial (ISO), la fístula entero-cutánea y la obstrucción por bridas/ adherencias representan el 76\% de las causas de reingreso por causa quirúrgica, la mayoría de manejo médico.

De acuerdo con el análisis de regresión logística realizado, el único factor de riesgo de una readmisión en el plazo de 30 días desde el egreso es haber sido reoperado por una complicación (re-operación no programada) durante la hospitalización índice (Tabla 6). Si se reconsideran algunos factores, incorporados en el test de regresión logística en forma dicotómica, como variables continuas, éstas tampoco alcanzan significación estadística como factores de riesgo para una readmisión no programada (la edad, la albúmina y el hematocrito preoperatorios, el tiempo operatorio y el tiempo de hospitalización índice obtienen un valor $p$ según el test de Mann-Whitney y el test de Wilcoxon de 0,676, 0,893, 0,596, 0,239 y 0,176 , respectivamente).

\section{Discusión}

La tasa de reingreso es un indicador complejo puesto que depende de múltiples factores, tales como los criterios de egreso empleados, la experiencia del equipo tratante, el acceso a centros de 
atención intermedios o de menor complejidad, la existencia de procesos instalados de atención de enfermería y/o hospitalización domiciliaria y el nivel sociocultural de los pacientes. Nos hemos inclinado a aceptar el plazo de 30 días desde el alta como límite para definir una readmisión no programada para poder comparar los resultados con otras series publicadas, considerando que estudios previos han establecido que en este período de 30 días se producen la gran mayoría (90\%) de los reingresos no planificados?.

En nuestra serie no hubo readmisiones luego de 15 días desde el alta y el $61 \%$ ocurre antes de la semana del egreso, lo que no elevó la TR global. En el estudio SEER mencionado, el 43,7\% de los reingresos ocurren en los primeros 7 días del egreso y sólo $12,7 \%$ ocurren después de 3 semanas $^{5}$. En esta serie de una institución pública con un equipo certificado y dedicado a la cirugía colorrectal, la tasa de reingreso global es $7 \%$, algo inferior a lo publicado en la literatura extranjera. Kiran publica la experiencia de la Cleveland Clinic, Ohio, USA con una tasa de $10 \%$, cifra que se eleva en los pacientes que requieren corticoides durante la hospitalización índice, concluyendo que la TR no es un indicador de calidad de la atención. En una serie posterior del mismo centro que compara dos grupos similares de 150 pacientes los autores concluyen que los predictores de reingreso luego de cirugía colorrectal son la enfermedad pulmonar obstructiva (EPOC), el tratamiento anticoagulante previo, el uso de esteroides y el egreso a una institución distinta que el hogar del paciente 7 .

Los factores predictivos de una re-hospitalización son motivo de controversia. En nuestra serie el único factor estadísticamente relevante fue el antecedente de una re-operación durante la cirugía índice, lo que apunta al impacto clínico que provoca en el paciente una reintervención no programada ${ }^{8}$. $\mathrm{O}^{\prime}$ Brien publica una TR de $10 \%$ en 820 pacientes consecutivos sometidos a cirugía laparoscópica colorrectal, confirmando que la EPOC y la enfermedad inflamatoria son factores de riesgo independientes de una readmisión 9 . Indirectamente los autores confirman que el egreso precoz asociado a la cirugía laparoscópica no eleva los índices de reingreso. Una serie de 351 pacientes sometidos a un cierre de una ostomía en la misma institución reporta una TR de $12,5 \%$, sugiriendo que es posible identificar a los pacientes en riesgo de sufrir un reingreso en forma prospectiva ${ }^{10}$. Para algunos autores, en cambio, no es posible predecir qué pacientes van a requerir una readmisión a partir de la evolución postoperatoria ${ }^{11}$. A pesar de lo controvertido del tema, una ley conocida como Programa de Reducción de los Reingresos, en la actualidad penaliza a los hospitales de USA con TR elevadas según el criterio del Medicare, sin distinguir en reingresos necesarios de los potencialmente evitables ${ }^{12}$. Estos últimos incluyen la deshidratación, la ISO superficial y la fiebre por una ITU que en nuestra experiencia representa el $66 \%$ de las causas de reingreso, dada la carencia de un manejo alternativo ambulatorio o de un centro intermedio de cuidados, lo que hace difícil comparar los resultados de este estudio con los publicados en el extranjero. En el estudio mencionado ${ }^{12}$, sobre un total de 3.536 casos de cirugía colorrectal (incluye urgencias y cirugía anal) los pacientes que reingresan son más ancianos, tienen más comorbilidades, fueron sometidos a cirugías más prolongadas y tuvieron una estadía hospitalaria más larga en la intervención índice, opuesto a lo encontrado en nuestra serie. Guinier, de la Asociación Francesa de Cirugía, en un universo de 1.421 sometidos a cirugía colorrectal obtiene una TR no programada de $7,5 \%$, señalando que de acuerdo al análisis multivariado de regresión logística los factores independientes asociados con el evento son la contaminación del campo operatorio, la duración de la cirugía, la necesidad de procedimientos quirúrgicos adicionales, una hemoglobina $<12 \mathrm{~g} / \mathrm{dl}$ y el hecho de no efectuar el test con aire de hermeticidad de la anastomosis colorrectal ${ }^{13}$.

En una revisión sistemática y meta-análisis de 60.131 reingresos, Li señala que la TR no programada fue $11 \%$ a los 30 días $^{14}$. Aunque no es sorprendente, los ancianos tuvieron una TR menor que los jóvenes, la principal causa de reingreso fue la obstrucción intestinal $(33,4 \%)$, seguido de la ISO $(15,7 \%)$ y el absceso intraabdominal $(12,6 \%)$, cifras similares a nuestra serie y otros estudios ${ }^{9,11}$. Los autores identifican varios factores de riesgo asociados a la edad, la capacidad funcional, la EPOC, comorbilidades múltiples y el egreso a una institución distinta del hogar del paciente. Un estudio efectuado en Inglaterra sobre un universo de 186.013 pacientes concluye que la TR es mayor en cirugía rectal que colónica $(9,4$ vs $7,6 \%, p<0,001)$ y que los predictores de un reingreso no programado son la resección de recto, la patología benigna, el paciente joven y la condición socio-económica baja ${ }^{15}$. Kelly en Irlanda analiza 8.197 pacientes sometidos a una resección colorrectal con una TR de 7,3\%, encontrando como único factor de riesgo de reingreso la estadía prolongada en la hospitalización índice ${ }^{16}$. La gran heterogeneidad de los estudios publicados podría, en parte, explicar resultados tan disímiles.

A pesar de que una readmisión se considera, y de hecho es, un hecho negativo en la evolución de un paciente, aparentemente no afecta los resultados finales de la intervención ni eleva la mortalidad global ${ }^{3,7,9}$, como ocurrió en nuestra serie. Desgraciadamente algunos estudios muestran que los costos 
adicionales asociados a un reingreso son considerables $^{6,12}$. Los estudios publicados muestran una TR variable $(7,5-15 \%)$ y factores predictivos extremadamente variados con resultados controversiales $\mathrm{u}$ opuestos, lo que hace difícil establecer el perfil del paciente con mayor riesgo de sufrir un reingreso en forma categórica. En nuestra serie no hemos encontrado diferencias significativas en la TR de acuerdo a factores propios del paciente (edad, género, grado de anemia, albúmina y comorbilidades), del tipo de patología (benigna/maligna) ni de los resultados de la operación índice (tiempo operatorio, estadía hospitalaria, ISO superficial, porcentaje de fístula) salvo la tasa de reoperación en la primera intervención, que en nuestra serie representa un factor predictivo independiente de reingreso. La cirugía laparoscópica, asociada a una menor estadía hospitalaria, muestra cifras de reingreso similares a la cirugía abierta 9 . Una TR alrededor del 10\% implica que los criterios de egreso utilizados son razonables, lo que obliga al equipo tratante a efectuar controles ambulatorios y telefónicos precoces, en ausencia de un protocolo de atención domiciliaria avanzada. En nuestra experiencia, el alta precoz en cirugía colorrectal electiva no es un factor de riesgo de un reingreso no programado. Mejorar estos índices probablemente implica implementar un soporte ambulatorio organizado del paciente quirúrgico, estrategia que se ha esbozado en nuestro hospital desde hace 2 años.

\section{Referencias}

1. Li LT, Mills WL, Gutiérrez AM, Herman LI, Berger DH, Naik AD. A patient-centered early warning system to prevent readmission after colorectal surgery: a national consensus using the Delphi method. J Am Coll Surg. 2013;216:210-6.

2. García-Botello S, Cánovas de Lucas R, Tornero C, Escamilla B, Espí-Macías A, Esclapez-Valero P y cols. Implantación de un protocolo de rehabilitación multimodal perioperatoria en cirugía colorrectal electiva. Estudio prospectivo, aleatorizado y controlado. Cir Esp. 2011;89:159-66.

3. Kiran RP, Delaney CP, Senagore AJ, Steel M, Garafalo T, Fazio VW. Outcomes and prediction of hospital readmission after intestinal surgery. J Am Coll Surg. 2004;198:877-83.

4. Hendren S, Morris AM, Zhang W, Dimick J. Early discharge and hospital readmission after colectomy for cancer. Dis Colon Rectum 2011;54:1362-7.

5. Schneider EB, Hyder O, Brooke BS, Efron J, Cameron $\mathrm{JL}$, Edil BH, et al. Patient readmission and mortality after colorectal surgery for colon cancer: impact of stay relative to other clinical factors. J Am Coll Surg 2012; 214: 390-8.

6. Wick EC, Shore AD, Hirose K, Ibrahim AM, Gearhart SL, Efron J, et al. Readmission rates and cost following colorectal surgery. Dis Colon Rectum 2011;54:1475-9.

7. Kariv Y, Wang W, Senagore AJ, Hammel JP, Fazio VW, Delaney CP. Multivariable analysis of factors associated with hospital readmission after intestinal surgery. Am J Surg. 2006;191:364-71.

8. Bannura G, Cumsille MA, Barrera A, Contreras J, Melo C, Soto D, Mansilla JA. Reoperaciones precoces en cirugía colorrectal. Análisis uni y multivariado de factores de riesgo. Rev Chil Cir. 2007;59:281-6.

9. O'Brien DP, Senagore A, Merlino J, Brady K, Delaney C. Predictors and outcome of readmission after laparoscopic intestinal surgery. World J Surg. 2007;31:2430-5.

10. Keller DS, Khorgami Z, Swendseid B, Khan S, Delaney $\mathrm{CP}$. Identifying causes for high readmission rates after stomal reversal. Surg Endosc. 2013;DOI 10.1007.

11. Azimuddin K, Rosen L, Reed JF, Stasik JJ, Riether RD, Khubcahndani IT. Readmissions after colorectal surgery cannot be predicted. Dis Colon Rectum 2001;44:942-6.

12. Keller DS, Swendseid B, Khorgami Z, Champagne BJ, Reynolds HL, Stein SL, et al. Predicting the unpredictable: comparing readmitted versus non-readmitted colorectal surgery patients. Am J Surg. 2014;207:346-51.

13. Guinier D, Mantion GA, Alves A, Kwiatkowski F, Slim $\mathrm{K}$, Panis Y. Risk factors of unplanned readmission after coorectal surgery: a prospective, multicenter study. Dis Colon Rectum 2007;50:1316-23.

14. Li LT, Mills WL, White DL, LI A, Gutiérrez AM, Berger DH, et al. Causes and prevalence of unplanned readmissions after colorectal surgery: a systematic review and meta-analysis. J Am Geriatr Soc. 2013;61:1175-81.

15. Faiz O, Haji A, Burns E, Bottle A, Kennedy R, Aylin P. Hospital stay amongst patients undergoing major elective colorectal surgery: predicting prolonged stay and readmissions in NHS hospitals. Colorectal Dis. 2011;13:816-22.

16. Kelly M, Sharp L, Dwane F, Kelleher T, Comber H. Factors predicting hospital length-of-stay and readmission after colorectal resection: a population-based study of elective and emergency admissions. MBC Health Serv Res. 2012;12:77. Doi:10.1186/1472-6963-12-77. 